\title{
Deep Brain Stimulation in Epilepsy: A Role for Modulation of the Mammillothalamic Tract in Seizure Control?
}

Frédéric L. W. V. J. Schaper, MD (10**§

Birgit R. Plantinga, $\mathrm{PhD}^{\ddagger \S}$ Albert J. Colon, MD, PhD ${ }^{\text {n }}$

G. Louis Wagner, $M^{\text {n } \|}$

Paul Boon, MD, PhD" $\|$ \#

Nadia Blom, MSc $^{\ddagger} \S$

Erik D. Gommer, PhD**

Govert Hoogland, $\mathrm{PhD}^{\ddagger \S \|}$

Linda Ackermans, MD, PhD ${ }^{\ddagger}$

Rob P. W. Rouhl, MD, PhD*§॥

Yasin Temel, MD, $\mathrm{PhD}^{\ddagger \S}$

*Department of Neurology, Maastricht University Medical' Center (MUMC+), Maastricht, The Netherlands; ${ }^{\ddagger}$ Department of Neurosurgery, Maastricht University Medical Center (MUMC+), Maastricht, The Netherlands; ${ }^{\S}$ School for Mental Health and Neuroscience (MHeNS), Maastricht University, Maastricht, The Netherlands; "Academic Center fo Epileptology Kempenhaeghe/Maastricht University Medical Center Heeze, The Netherlands; "Academic Center for Epileptology Kempenhaeghe/Maastricht University Medical Center, Maastricht, The Netherlands; "Department of Neurology, University Hospital Ghent Ghent, Belgium; ***Department of Clinical Neurophysiology, Maastrich University Medical Center, Maastricht The Netherlands

\section{Correspondence:}

Frédéric L. W. V. J. Schaper, MD, Department of Neurosurgery,

Maastricht University Medical Center, Universiteitsssingel 50,

6229 ER Maastricht, The Netherlands. Email: f.schaper@maastrichtuniversity.nl Twitter: @fredschaper3

Received, June 24, 2019.

Accepted, February 16, 2020

Published Online, May 18, 2020.

(C) The Author(s) 2020. Published by Oxford University Press on behalf of Congress of Neurological Surgeons. This is an Open Access article distributed under the terms of the Creative Commons Attribution Non-Commercial License (http://creativecommons.org/ licenses/by-nc/ 4.0/), which permits non-commercial reuse, distribution, and reproduction in any medium, provided the original work is properly cited. For commercial re-use, please contact journals.permissions@oup.com

BACKGROUND: Deep brain stimulation of the anterior nucleus of the thalamus (ANT-DBS) can improve seizure control for patients with drug-resistant epilepsy (DRE). Yet, one cannot overlook the high discrepancy in efficacy among patients, possibly resulting from differences in stimulation site.

OBJECTIVE: To test the hypothesis that stimulation at the junction of the ANT and mammillothalamic tract (ANT-MTT junction) increases seizure control.

METHODS: The relationship between seizure control and the location of the active contacts to the ANT-MTT junction was investigated in 20 patients treated with ANT-DBS for DRE. Coordinates and Euclidean distance of the active contacts relative to the ANT-MTT junction were calculated and related to seizure control. Stimulation sites were mapped by modelling the volume of tissue activation (VTA) and generating stimulation heat maps. RESULTS: After $1 \mathrm{yr}$ of stimulation, patients had a median $46 \%$ reduction in total seizure frequency, $50 \%$ were responders, and $20 \%$ of patients were seizure-free. The Euclidean distance of the active contacts to the ANT-MTT junction correlates to change in seizure frequency $\left(r^{2}=0.24, P=.01\right)$ and is $\sim 30 \%$ smaller $(P=.015)$ in responders than in nonresponders. VTA models and stimulation heat maps indicate a hot-spot at the ANT-MTT junction for responders, whereas non-responders had no evident hot-spot.

CONCLUSION: Stimulation at the ANT-MTT junction correlates to increased seizure control. Our findings suggest a relationship between the stimulation site and therapy response in ANT-DBS for epilepsy with a potential role for the MTT. DBS directed at white matter merits further exploration for the treatment of epilepsy.

KEY WORDS: Neuromodulation, DRE, Drug-resistant epilepsy, Thalamus, Mammillothalamic tract, White matter stimulation

Neurosurgery 87:602-610, 2020

DOI:10.1093/neuros/nyaa141

www.neurosurgery-online.com

D eep brain stimulation of the anterior nucleus of the thalamus (ANT-DBS) has recently been approved by the U.S. Food and Drug Administration (FDA) for

ABBREVIATIONS: ANT, anterior nucleus of the thalamus; DBS, deep brain stimulation; DRE, drugresistant epilepsy; ICC, intraclass correlation coefficients; MCP, midcommissural point; MTT, mammillothalamic tract; SANTE, stimulation of the anterior nucleus of the thalamus for epilepsy; VTA, volume of tissue activation

Neurosurgery Speaks! Audio abstracts available for this article at www.neurosurgery-online.com.

Supplemental digital content is available for this article at www.neurosurgery-online.com.

CNS Spotlight available at cns.org/spotlight. the treatment of patients with drug-resistant epilepsy (DRE) when resective procedures or less invasive neuromodulation therapies are not possible or have failed. The stimulation of the anterior nucleus of the thalamus for epilepsy (SANTE) trial demonstrated that bilateral thalamic stimulation in drug-resistant focal epilepsy is a safe procedure that reduces shortand long-term seizure frequency and significantly improves well-being. ${ }^{1,2}$ While several cohorts following the SANTE trial confirm these findings with mean reported responder rates across studies approximating 50\% after $1 \mathrm{yr}$ of ANT-DBS, ${ }^{3-6}$ the degree of seizure control can vary highly between patients. ${ }^{7}$ Knowledge obtained from DBS in movement disorders suggests that patient selection ${ }^{8}$ and electrode placement ${ }^{9}$ are important factors for 
predicting clinical outcome. As such, suggested denominators for seizure control by DBS are patient characteristics, such as the location of seizure onset, and the stimulation site. ${ }^{4,6}$ Furthermore, data from the SANTE trial indicate that DBS leads were not consistently placed within the ANT, ${ }^{10}$ yet effective stimulation with contacts outside the ANT has been reported. ${ }^{11}$ Hence, the optimal stimulation site is debated.

DBS lead placement within the ANT is currently performed by direct neurosurgical targeting, ${ }^{12,13}$ in which the mammillothalamic tract (MTT) functions as a key anatomical landmark. ${ }^{14,15}$ The MTT is a prominent white matter bundle that arises from the mammillary bodies and ends in the medio-ventral part of the ANT, where it joins the internal and external lamina of the thalamus, also known as the ANT-MTT junction. Within the circuit of Papez, the ANT receives major afferent input from the hippocampal formation through the MTT next to its reciprocal cortical connections through thalamic radiations ${ }^{16}$ and thalamocingulate fibers. ${ }^{17}$ While the mechanism of action still remains elusive and it is unclear to what degree different brain networks and fiber tracts are stimulated, ANT-DBS is speculated to halt seizure propagation and/or modulate epileptogenic foci through its connections to the circuit of Papez. ${ }^{18}$ The significance of the circuit of Papez as a potential seizure circuit is exemplified by depth recordings in humans ${ }^{11,19,20}$ and lesion studies in animals, ${ }^{21,22}$ Accordingly, the varied effects of ANTDBS possibly relate to unsuccessful stimulation of the $\mathrm{MTT}^{23}$ to achieve seizure circuit control.

In this study, we hypothesized that stimulation of the ANTMTT junction increases seizure control. We performed an independent, clinical-outcome blinded analysis of the active contacts in our ANT-DBS patient cohort to investigate the relationship between stimulation site and seizure control in DRE.

\section{METHODS}

\section{Patients, Surgery, and DBS}

We included all patients who qualified for on-label DBS treatment for DRE. ${ }^{1,13}$ Patients were assessed by an epilepsy expert panel, were not eligible for resective surgery or did not respond to previous resective procedures or vagal nerve stimulation. Details of our DBS surgery for epilepsy are described elsewhere. ${ }^{13}$ In short, DBS surgery was performed under general anesthesia guided by microelectrode recordings along an extraventricular surgical trajectory (Figure 1) and 3389 leads (Medtronic, Dublin, Ireland) were bilaterally implanted at the ANT. Following evaluation of the DBS lead position for minor postsurgical movement by the neurosurgeon, the pulse generator was turned on 6 weeks after surgery with the following stimulation parameters: frequency of $145 \mathrm{~Hz}$, intensity of $5 \mathrm{~V}$, pulse-width of $90 \mu \mathrm{s},{ }^{1}$ and thereafter adjusted at the discretion of the epileptologist. Therapy response was assessed at 1-yr follow-up after start of stimulation. We considered patients with $\geq 50 \%$ reduction in seizure frequency compared to baseline as responders and patients with $<50 \%$ reduction as non-responders.

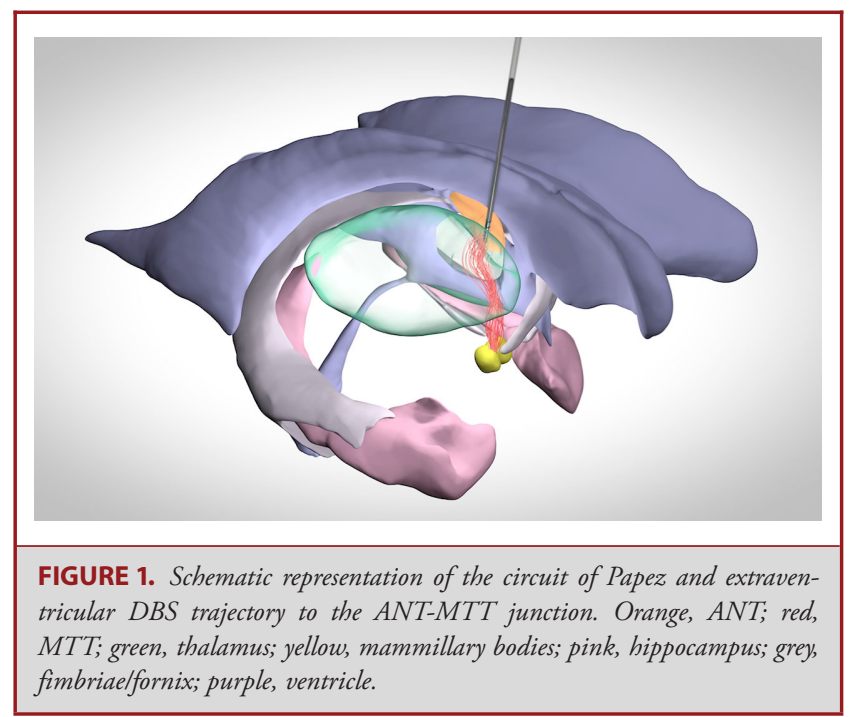

\section{Ethical Statement}

The work described was conducted in accordance with the Declaration of Helsinki. Approval by the institutional review board and patient consent were not required as the present study has no obligations to the Dutch Act of Scientific Research in Humans.

\section{Imaging}

All subjects had a preoperative 3T or 1.5T MRI (Philips, Eindhoven, The Netherlands) in case of an implanted vagal nerve stimulator. The sequences used were a 3D T1 with gadolinium (voxel sizes: $1 \times 1 \times 1 \mathrm{~mm}$, TE/TR of 3.7/8.1 ms), axial T2 (voxel sizes: $0.45 \times 0.45 \times 2 \mathrm{~mm}$, TE/TR of $80 \mathrm{~ms} / 8264 \mathrm{~ms}$ ), and a T1 inversion recovery (voxel sizes: $0.34 \times 0.34 \times 2 \mathrm{~mm}$, TE/TR/TI of 10/7362/400 ms). Postoperative CT (Siemens, Erlangen, Germany) or 1.5T T1 (voxel sizes: $1 \times 1 \times 1 \mathrm{~mm}$, TE/TR of 4.6/9.3 ms) MRI was performed for DBS lead localization in the week following DBS surgery.

\section{ANT-MTT Junction}

Preoperative and postoperative images were fused in individual stereotactic space, also termed native space, on the Medtronic Stealthstation S7, and the midcommissural point (MCP) was identified by a neurosurgeon (YT). Coordinates relative to MCP were assessed for the ANT-MTT junction (Figure 2) for each hemisphere by 2 observers independently (FS and YT). Both observers were blinded to clinical outcome. Definite coordinates in lateral $(x)$, anterior $(y)$, and superior $(z)$ directions were defined by the mean of the coordinates that were given by the two observers. In case the observers disagreed $\geq 1 \mathrm{~mm}$ in either the $x, y$, or $z$ direction in the first observation, the final coordinates were based on their consensus in a second observation.

\section{Active Contact Location}

The locations of the active contacts were analyzed as previously published. ${ }^{24,25}$ In short, the active contact coordinates were calculated from the coordinates of the lead tip as defined on fused pre- and postoperative images, a reference point within the trajectory and the 

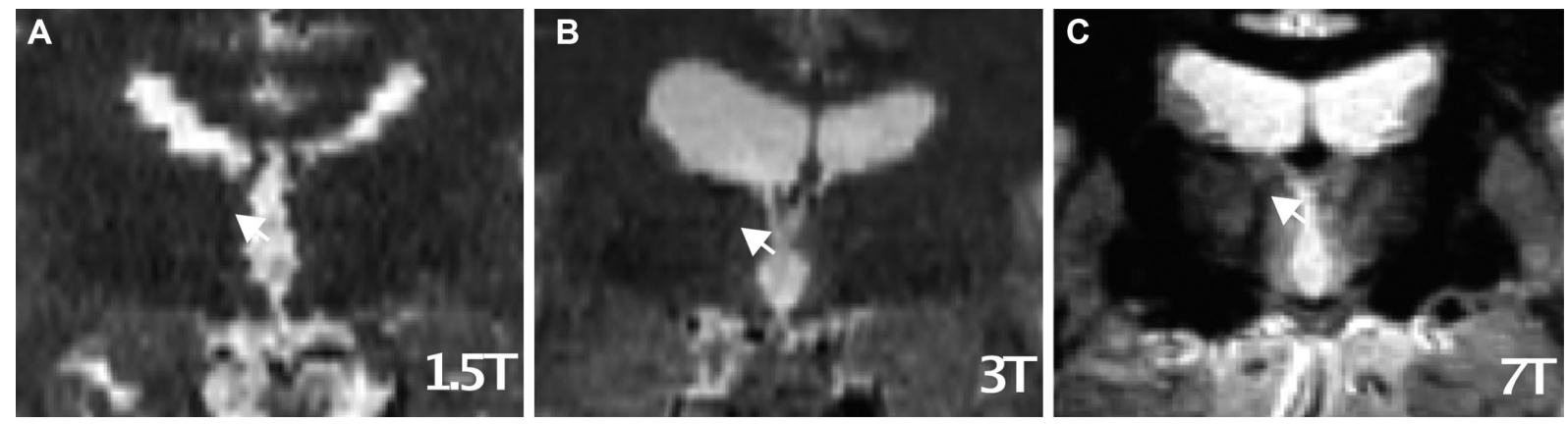

FIGURE 2. The ANT-MTT junction (arrow) at $1.5 T \mathrm{~A}, 3 T \mathrm{~B}$, and $7 T \mathrm{C} M R I$ field strengths.

interelectrode distance. For a bipolar contact configuration, the coordinates of the point halfway along the vector in between the cathode and anode were chosen. We analysed the distance of the active contact to the ANT-MTT junction in $x, y$, and $z$ directions in native space and calculated the shortest distance, also known as the Euclidean distance. Using MATLAB (R2015a, MathWorks, Natick, Massachusetts), the locations of the active contacts were plotted in a common space, henceforth called ANT-MTT normalized space. The common origin in ANT-MTT normalized space $[x=0, y=0, z=0]$ was set at the coordinates of the ANT-MTT junction in native space. Locations of the active contacts are presented as coordinates relative to this point of origin for each individual patient. The rate of the active contacts located within the ANT was assessed according to Lehtimäki et al $2018 .^{26}$

\section{Volume of Tissue Activation}

Volume of tissue activation (VTA) for both monopolar and bipolar contact configurations was modelled according to the methods described by Chaturvedi et al 2013. ${ }^{27}$ In short, the spatial extent of axonal activation was characterized by artificial neural networks based on finite element models of the electrical fields generated by the DBS lead with patient specific stimulation parameters. VTAs were subsequently plotted around the active contact coordinates in ANT-MTT normalized space. To visualize a common volume of tissue activated within each group, a stimulation heat map was generated for responders and non-responders using an activation score as described by Chueng et al. ${ }^{28}$ These were subsequently superimposed on the Mai atlas $3 \mathrm{rd} \mathrm{edition}^{29}$ to visualize the hot-spot of stimulation (intersection of VTAs with the highest activation score) in anatomic space.

\section{Statistical Analysis}

Intraclass correlation coefficients (ICC) were calculated to evaluate inter-observer reliability, and Pearson correlation was used to investigate the relationship between seizure control and the Euclidean distance of the active contact to the ANT-MTT junction. Coordinates relative to MCP or ANT-MTT junction and Euclidean distances were compared between groups by a Mann-Whitney $\underline{\mathrm{U}}$ test. Patient characteristic were compared between groups using a Mann-Whitney $U$ or Chi-square test, as appropriate. $P$-values $<.05$ were considered statistically significant.

\section{RESULTS}

\section{Patients and Seizure Control}

We included 20 patients with 1 -yr follow-up of stimulation. We classified 10 patients as responders and 10 patients as non-responders, resulting in a responder rate of $50 \%$ with a median $46 \%$ reduction in total seizure frequency, and $20 \%$

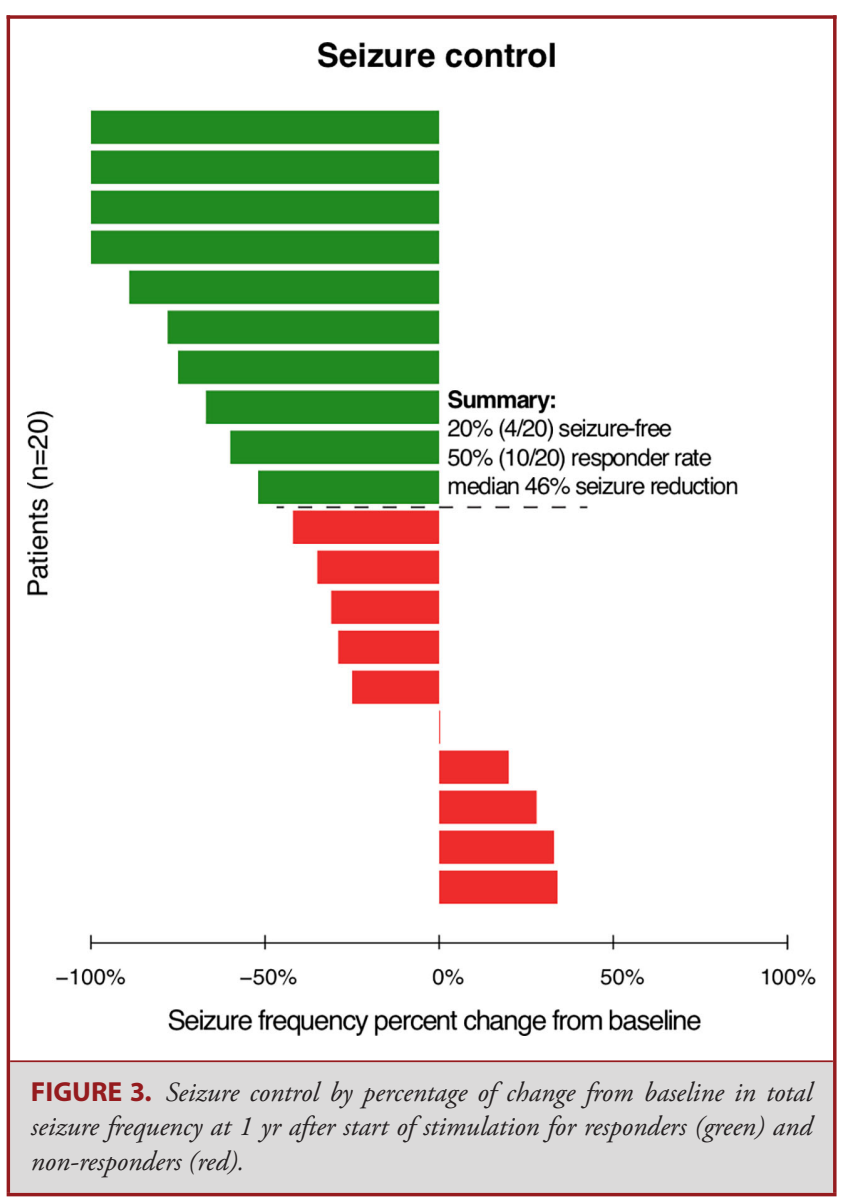


TABLE. ANT-MTT Junction and Active Contact Locations

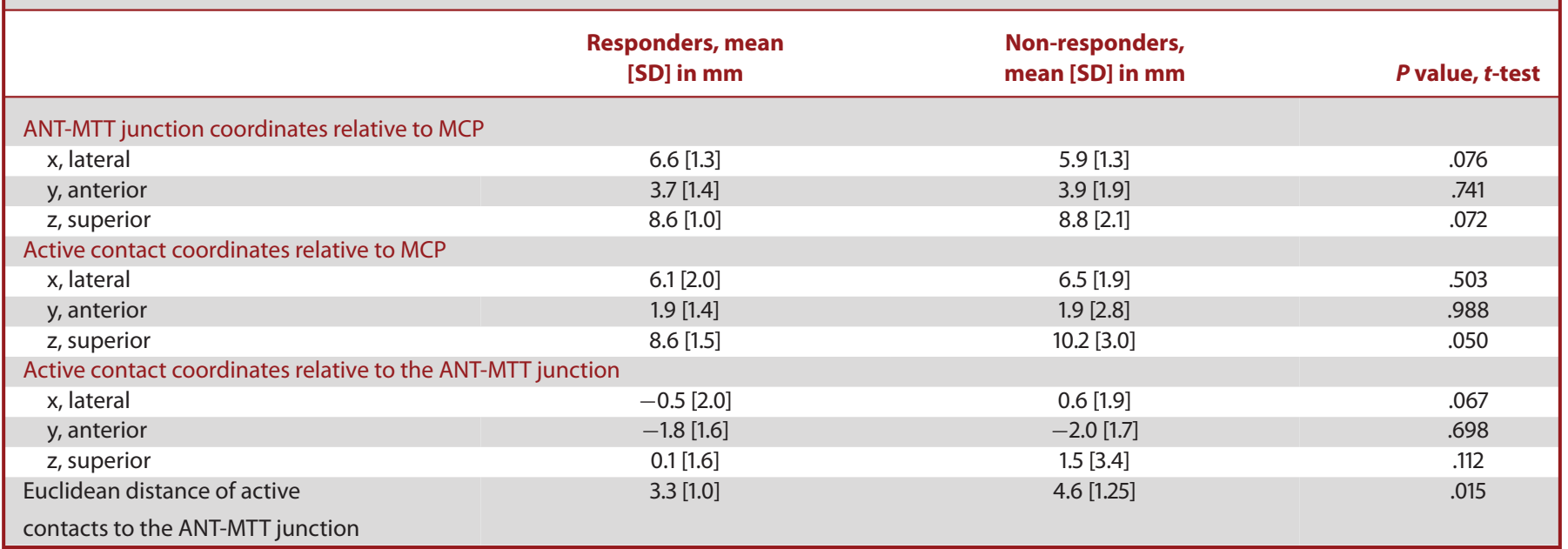

of patients were seizure-free (Figure 3). Group characteristics and individual patient characteristics can be found in Tables, Supplemental Digital Contents 1 and 2 . No significant differences were found between responders and non-responders for possible confounders such as age, epilepsy duration, the suspected seizure-onset zone or prior therapy. Of note, a seizure-onset zone in the temporal lobe was more prevalent in responders (4/10) compared to non-responders (2/10), but extratemporal seizure onset was similar between groups (5/10). Multifocal seizure onset was more prevalent in non-responders $(3 / 10)$ than in responders $(1 / 10)$.

\section{ANT-MTT Junction}

For every individual patient, the ANT-MTT junction was identified on preoperative MR images by two independent observers. The inter-observer reliability was excellent (ICC $=0.99, P<.001$ ) for the first (independent) observations of the ANT-MTT junction. In 30/40 of these observations, the coordinates for the ANT-MTT junction by the two observers differed $<1 \mathrm{~mm}$ in all directions and thus the mean coordinates of the first observation were used in further analysis. In 10/40 of first observations, the coordinates of the ANT-MTT junction by the 2 observers differed $\geq 1 \mathrm{~mm}$ in either the $x, y$, or $z$ direction and, therefore, a consensus was reached in a second observation and used in the final analysis. The definite mean [SD] ANT-MTT junction coordinates relative to MCP for the total ANT-DBS population were $x=6.2 \mathrm{~mm}[1.3], y=3.8 \mathrm{~mm}$ [1.7], and $z=8.7 \mathrm{~mm} \mathrm{[1.6],} \mathrm{which} \mathrm{represent} \mathrm{the} \mathrm{stereotactic}$ coordinates for indirect targeting of the ANT-MTT junction. These ANT-MTT junction coordinates considerably differ from the ANT coordinates $(x=5-6 \mathrm{~mm}, y=0-2 \mathrm{~mm}$, $z=12 \mathrm{~mm}$ ) commonly used for indirect targeting in ANT-DBS.

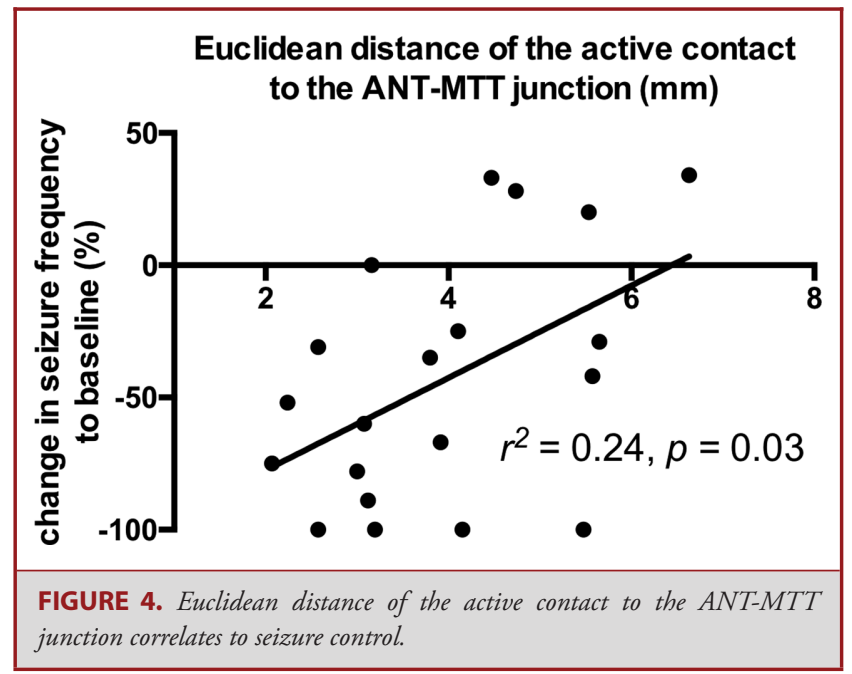

\section{Active Contact Location}

Active contact coordinates relative to MCP and the ANTMTT junction are presented in Table. The active contacts of responders were localized more medio-inferior towards the ANT-MTT junction compared to non-responders, which were localized more latero-superior and latero-inferior. The Euclidean distance of the active contact to the ANT-MTT junction is $29 \%$ smaller $(P=.015)$ in responders (mean [SD]: $3.3 \mathrm{~mm}[1.0])$ compared to non-responders (mean [SD]: $4.6 \mathrm{~mm}[1.25]$ ). The Euclidean distance correlated with change in seizure frequency after 1 yr of DBS $\left(r^{2}=0.24, P=.03\right)$, implicating active contacts located closer to the ANT-MTT junction are more likely to reduce seizure frequency (Figure 4). Of all 44 active contacts, 45\% (20/44) were placed within the ANT and 55\% outside the ANT (24/44). Contacts outside the ANT were situated in other 


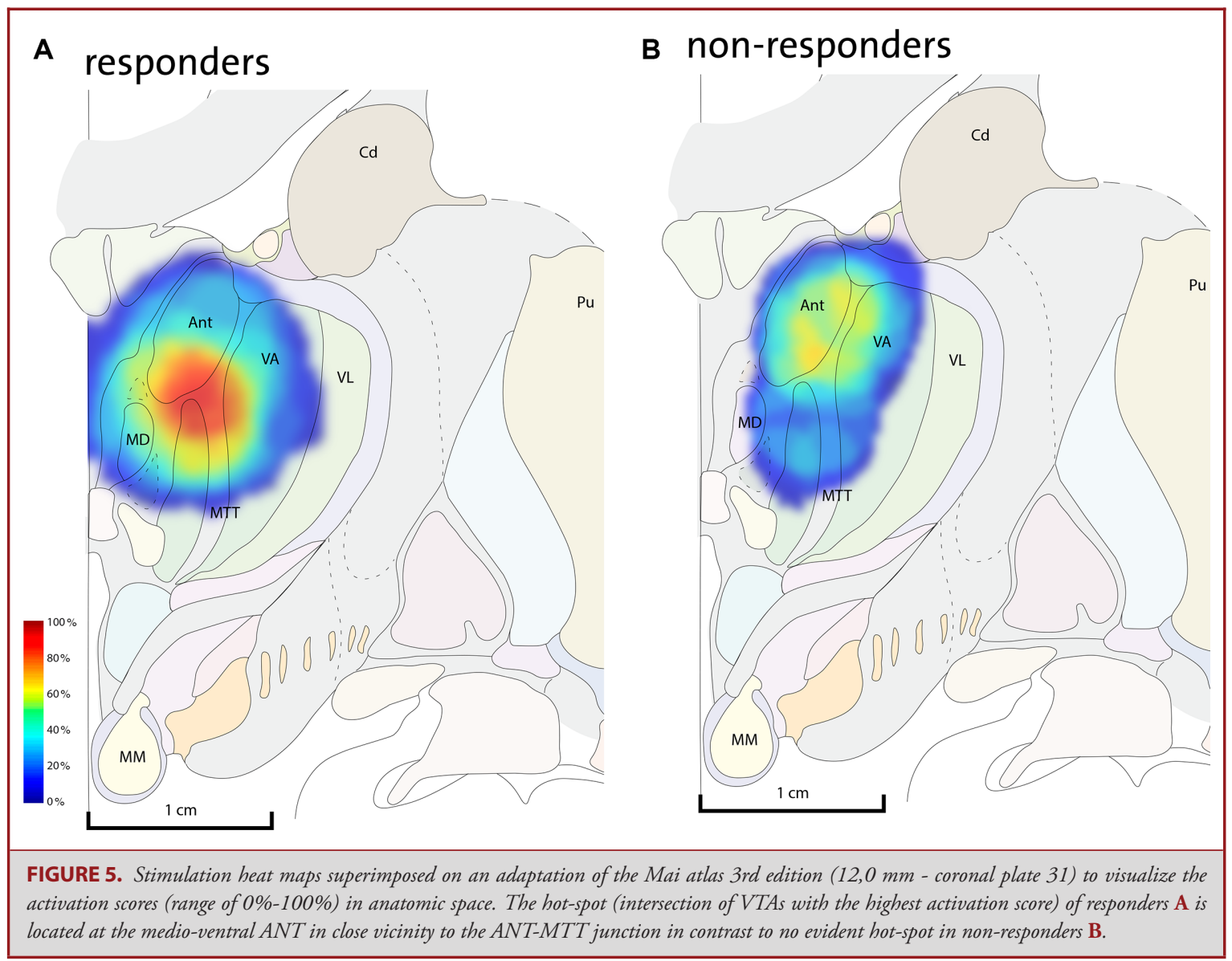

thalamic subnuclei (eg, mediodorsal and ventral anterior nucleus) or white matter structures (eg, MTT and medullary lamina of the thalamus). Within the 2 groups, $36 \%$ (8/22) of active contacts of responders and $55 \%(12 / 22)$ of active contacts of non-responders were placed within the ANT.

\section{VTA}

Stimulation parameters at 1-yr follow-up entailed a mean amplitude [SD] of $5.6 \mathrm{~V}$ [0.4] with a pulse width of $90 \mu \mathrm{s}$, frequency of $145 \mathrm{~Hz}$, and a stimulation-cycling mode of $1 \mathrm{~min}$ on and 5 min off in all subjects. Eighteen subjects received bilateral monopolar stimulation and 2 subjects received bilateral bipolar stimulation considering side effects, namely irritability and sleep problems. Information on individual subjects' active contacts is included in Table, Supplemental Digital Content 2. VTA models were calculated from the patients' individual stimulation parameters, plotted in ANT-MTT normalized space, and superimposed on the Mai atlas ${ }^{29}$ (Figure 5). The stimulation hot-spot of responders was at the medio-ventral ANT in close vicinity to the ANT-MTT junction. In non-responders, there was no evident stimulation hot-spot as the VTAs were heterogeneously distributed either at the dorsal ANT or ventral anterior nucleus (see Figure, Supplemental Digital Content 3 for heat maps in coronal and sagittal views).

\section{DISCUSSION}

In this study, we investigated the relationship between stimulation site and therapy response to ANT-DBS in 20 patients with DRE by analyzing the locations of the active contacts and VTA in respect to the ANT-MTT junction. Our results indicate that the ANT-MTT junction can be used as an anatomical landmark for neurosurgical targeting in ANT-DBS and is identified with excellent interobserver reliability. Active contacts more closely located to the ANT-MTT junction were associated with increased seizure control. The stimulation hot-spot of responders was at the medio-ventral ANT in high vicinity to the ANT-MTT junction in contrast to no evident hot-spot in non-responders. Accordingly, the ANT-MTT junction is not only an anatomical landmark for direct neurosurgical targeting, but also a potential stimulation site for increased seizure control. Although not investigated in this study, neurosurgeons could revise the lead locations and 
neurologists could reprogram the pulse generator of nonresponders to include stimulation of the ANT-MTT junction and optimize seizure control in patients with previously implanted DBS leads. Co-stimulation of white matter tracts may play a fundamental role in the mechanism of action of seizure control by ANT-DBS and warrants further study.

Historically, DBS is directed at grey matter and is thought to mimic a reversible, local lesioning effect. Recently, experience in movement disorders and psychiatric conditions has shown that DBS can have distant network effects, can modulate neurotransmitter release, induces neuroplasticity, and might even have permanent structural effects leading to disease course modification. ${ }^{30}$ These global effects cannot be explained by a mere local lesioning effect and, consequently, researchers have concentrated on effects on brain states, modulated in part by white matter tracts. A recent promising example of neuromodulation specifically directed at white matter is the investigation of DBS of the fornix for dementia-related disorders. ${ }^{31}$ Yet, in the epilepsy field, targeting fiber tracts has long shown to have striking effects, as callosotomy and VNS are well-established therapies for selected patients with DRE. Interestingly, in the first fundamental experimental studies suggesting involvement of the circuit of Papez in seizure control, Mirski et al ${ }^{21}$ revealed that lesioning or electrical stimulation of the MTT can protect against chemically induced seizures in guinea pigs. Consequently, interruption of this key connection by high frequency stimulation of the ANT had similar results in rats. ${ }^{32,33}$ These experimental animal studies, the pioneering human pilots of Cooper and Upton ${ }^{34}$ in the $1980 \mathrm{~s}$, along with several case series ${ }^{35,36}$ culminated in to the well-known investigation of ANT-DBS for the treatment of DRE by the SANTE study group.

\section{Effective Stimulation Sites in ANT-DBS for Epilepsy}

The SANTE study reported a median $56 \%$ reduction in seizure frequency compared to baseline after $2 \mathrm{yr}$ with a $54 \%$ responder rate and a $69 \%$ seizure reduction with a $68 \%$ responder rate after 5 yr. $^{1,2}$ A post hoc analysis revealed that DBS lead placements were not always within the ANT. Henceforth, there is only scarce data available on the relation between the location of the active contacts and clinical outcome in ANT-DBS. A study by Lehtimäki et $\mathrm{al}^{4}$ made an in-depth analysis of lead placement and location of the active contacts in their ANT-DBS cohort of 15 patients. Similar to the approach used here, they manually defined the borders of the ANT in native space and constructed an ANT-normalized coordinate system. Coupling the individual active contacts to therapy response, they found that responding contacts were located at the anterior aspect of the ANT, anterodorsal to the ANT-MTT junction, compared to a slightly more postero-ventral localization in our study. Krishna et $\mathrm{al}^{6}$ report on the locations of the active contacts and VTA of 7 responders to ANT-DBS in Montreal Neurological Institute (MNI) space. In line with our results, the stimulation hot-spot of responders was at the ANT-MTT junction. Contrary to our study, non-

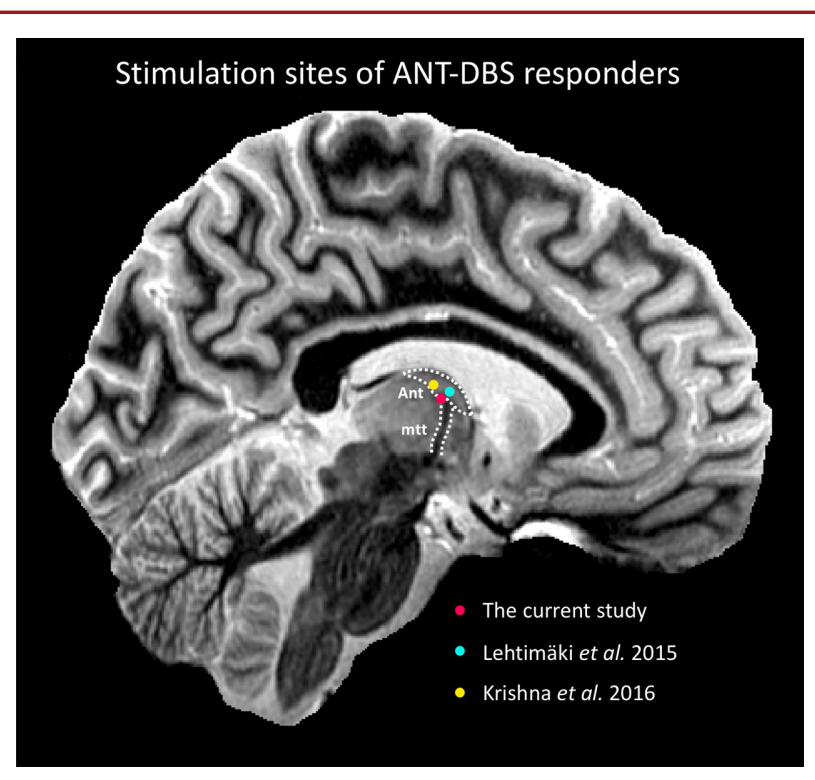

FIGURE 6. Visual representation of the stimulation sites of ANT-DBS responders in the current study and published studies, overlaid on a sagittal section of a $7 T M R$ image. The sequence used was a T1 white-matternulled MPRAGE ${ }^{51}$ (voxel size: $0.8 \times 0.8 \times 0.8 \mathrm{~mm}$, TE/TR/TI of 3.3/4.5/617 ms) obtained from a healthy control using a 7 T magnet (Siemens, Erlangen, Germany) and a 32-channel head coil (Nova Medical, Wilmington, Massachusetts) at the Maastricht Brain Imaging Centre. Abbreviations: ANT, anterior nucleus of the thalamus; MTT, mammillothalamic tract.

responders were not included in this analysis and a link between therapy response and stimulation site could therefore not be made. Our study reports on the clinical outcome of an extraventricular neurosurgical approach to the ANT and contributes to the current definition of effective stimulation sites in ANTDBS for epilepsy. In summary, we found that an extraventricular trajectory to the ANT results in similar (short-term) clinical outcome as reported in the SANTE trial, that the ANT-MTT junction can be identified with excellent inter-observer reliability, the active contact locations and stimulation hot-spots differ between responders and non-responders and that stimulation of the ANT-MTT junction correlates to increased seizure control.

\section{Future Perspectives in ANT-DBS Targeting and Stimulation}

Neurosurgeons commonly use frontal transventricular and extraventricular approaches in ANT-DBS targeting, which are both safe and well tolerated. The current study suggests that the location of the effective stimulation site is similar for the transventricular and extraventricular neurosurgical approach (Figure 6). Although a transventricular trajectory is more likely to place the contacts within the ANT due to its perpendicular approach and additionally allows for more superior stimulation in the ANT, the ANT-MTT junction can be stimulated by both 
trajectories. $^{26}$ A novel posterior parietal extraventricular trajectory ${ }^{37}$ has even been proposed recently, which is conventionally used for shunt surgeries. High accuracy (90\%) for placing contacts into the ANT was found with this approach leveraging the MTT junction as an anatomical landmark. ${ }^{38}$ Future studies comparing lead placement, stimulation sites, and clinical outcome of patients with different surgical trajectories will shed more light on the optimal surgical approach and stimulation site in ANT-DBS for epilepsy.

Considering the current available Level 3 evidence on stimulation sites in ANT-DBS, we advocate planning a neurosurgical trajectory to target the ANT-MTT junction and programming the pulse generator to stimulate this region. Given fibers can also be stimulated by lower frequencies, ${ }^{39}$ low-frequency stimulation could be an alternative effective stimulation paradigm in ANT-DBS for epilepsy, as supported by experimental animal studies. ${ }^{37,40}$ DBS directed at fiber tracts additionally vows to elongate battery life and decrease stimulation-induced side effects. Stimulation of a small population of axons could modulate a large population of distant (epileptic) neurons, ${ }^{41}$ thus supporting the clinical use of lower stimulation intensities. Ultra-high-field $\mathrm{MRI}^{42}$ and tractography based neurosurgical targeting methods ${ }^{43}$ could facilitate distinct stimulation of the MTT (see Figure, Supplemental Digital Content 4 for tractography of the MTT) or other fiber tracts to achieve seizure control for diverse forms of epilepsy. Potential white matter targets derived from the literature include the MTT ${ }^{44}$ thalamocingulate tract, ${ }^{45}$ corpus callosum, ${ }^{46}$ fornix ${ }^{47}$ cerebellothalamic, ${ }^{48}$ and pallidothalamic ${ }^{49}$ tracts. We anticipate renewed scientific interest and clinical exploration of white matter tract stimulation in DBS for epilepsy.

\section{Limitations}

The main limitations of the present study are fourfold. First, the number of patients included is low, resulting in a limited power to detect significant changes. Yet, similar studies on anatomical localization of DBS electrodes have used comparable sample sizes. ${ }^{4,28}$ The sample size here is the largest described yet for ANT-DBS and is thus representative, considering the currently scarce available evidence. Second, we did not use Medtronic Suretune, the commercial software tool that is aimed at localizing DBS leads in respect to an atlas or manual segmentations of grey matter nuclei. Due to the high interindividual and interhemispheric variability in (mammillo)thalamic anatomy ${ }^{10,14,50}$ and low MR contrast between the thalamic subnuclei $^{51}$, we instead chose to localize the leads to a patientspecific anatomical landmark (the ANT-MTT junction) using the Medtronic Stealthstation surgical navigation system. Subsequently, a normalized space for group analysis was constructed similarly to published studies. ${ }^{4,25}$ Inherently to a study on DBS lead localizations, there are possible minimal inaccuracies of image registration and active contact localization. Third, considering the VTA model is designed to estimate the activation of largediameter axons $(5.7 \mu \mathrm{m})$, the current predictions represent an overestimation of the spatial extent of stimulation and thus a "worst-case" scenario. ${ }^{27}$ Fourth, although not statistically significant in our cohort, confounding by patient demographics, location of the suspected seizure-onset zone, prior epilepsy surgery, or VNS cannot be excluded due to the retrospective nature of the study. The results of our study are correlative, and the importance of these potential confounding variables is still unknown. Hence, our results should be interpreted with caution. Due to the inclusion criteria of the current CE mark of ANT-DBS, the patient population of our cohort is typically heterogeneous. Future studies with larger and more homogeneous patient populations should replicate our results and investigate stimulation hot-spots for different seizure types and seizure-onset zones to move towards seizure circuit and patient-tailored DBS in epilepsy.

\section{CONCLUSION}

Stimulation of the ANT-MTT junction correlates to increased seizure control. Our findings suggest a relationship between stimulation site and therapy response in ANT-DBS for DRE with a potential role for modulation of the MTT. DBS directed at white matter merits further exploration for the treatment of epilepsy.

\section{Disclosures}

Dr Colon has received speaker honoraria from Medtronic. The other authors have no personal, financial, or institutional interest in any of the drugs, materials, or devices described in this article.

\section{REFERENCES}

1. Fisher R, Salanova V, Witt T, et al. Electrical stimulation of the anterior nucleus of thalamus for treatment of refractory epilepsy. Epilepsia 2010;51(5):899-908.

2. Salanova V, Witt T, Worth R, et al. Long-term efficacy and safety of thalamic stimulation for drug-resistant partial epilepsy. Neurology. 2015;84(10):1017-1025.

3. Lee KJ, Shon Y-M, Cho CB. Long-term outcome of anterior thalamic nucleus stimulation for intractable epilepsy. Stereotact Funct Neurosurg. 2012;90(6):379385.

4. Lehtimäki K, Möttönen T, Järventausta K, et al. Outcome based definition of the anterior thalamic deep brain stimulation target in refractory epilepsy. Brain Stimul. 2016;9(2):268-275.

5. Herrman H, Egge A, Konglund AE, Ramm-Pettersen J, Dietrichs E, Taubøll E. Anterior thalamic deep brain stimulation in refractory epilepsy: a randomized, double-blinded study. Acta Neurol Scand. 2019;139(3):294-304.

6. Krishna V, King NKK, Sammartino F, et al. Anterior nucleus deep brain stimulation for refractory epilepsy. Neurosurgery. 2016;78(6):802-811.

7. Bouwens van der Vlis TAM, Schijns OEMG, Schaper FLWVJ, et al. Deep brain stimulation of the anterior nucleus of the thalamus for DRE. Neurosurg Rev. 2018;12(1):1571-1510.

8. Kleiner-Fisman G, Herzog J, Fisman DN, et al. Subthalamic nucleus deep brain stimulation: summary and meta-analysis of outcomes. Mov Disord. 2006;21(S14):S290-S304.

9. Welter M-L, Schüpbach M, Czernecki V, et al. Optimal target localization for subthalamic stimulation in patients with parkinson disease. Neurology. 2014;82(15):1352-1361.

10. Wu C, D'Haese P-F, Pallavaram S, et al. Variations in thalamic anatomy affect targeting in deep brain stimulation for epilepsy. Stereotact Funct Neurosurg. 2016;94(6):387-396 
11. Osorio I, Frei MG, Lozano AM, et al. Subcortical (thalamic) automated seizure detection: a new option for contingent therapy delivery. Epilepsia. 2015;56(10): e156-e160.

12. Buentjen L, Kopitzki K, Schmitt FC, et al. Direct targeting of the thalamic anteroventral nucleus for deep brain stimulation by T1-weighted magnetic resonance imaging at 3 T. Stereotact Funct Neurosurg. 2014;92(1):25-30.

13. Schaper FLWVJ, Zhao Y, Janssen MLF, et al. Single-Cell recordings to target the anterior nucleus of the thalamus in deep brain stimulation for patients with refractory epilepsy. Int J Neur Syst. 2019;29(4):1850012.

14. Jiltsova E, Möttönen T, Fahlström M, et al. Imaging of anterior nucleus of thalamus using 1.5t mri for deep brain stimulation targeting in refractory epilepsy. Neuromodulation: Technology at the Neural Interface. 2016;19(8):812-817.

15. Möttönen T, Katisko J, Haapasalo J, et al. Defining the anterior nucleus of the thalamus (ANT) as a deep brain stimulation target in refractory epilepsy: delineation using $3 \hat{A}$ T MRI and intraoperative microelectrode recording. NeuroImage: Clinical. 2015;7:823-829.

16. Child ND, Benarroch EE. Anterior nucleus of the thalamus: functional organization and clinical implications. Neurology. 2013;81(21):1869-1876.

17. Weininger J, Roman E, Tierney P, et al. Papez's forgotten tract: 80 years of unreconciled findings concerning the thalamocingulate tract. Front Neuroanat. 2019;13:14

18. Laxpati NG, Kasoff WS, Gross RE. Deep brain stimulation for the treatment of epilepsy: circuits, targets, and trials. Neurotherapeutics. 2014;11(3):508-526.

19. Sweeney-Reed CM, Lee H, Rampp S, et al. Thalamic interictal epileptiform discharges in deep brain $\hat{A}$ stimulated epilepsy patients. I Neurol. 2016;263(10):2120-2126.

20. van Rijckevorsel K, Abu Serieh B, de Tourtchaninoff M, et al. Deep EEG recordings of the mammillary body in epilepsy patients. Epilepsia. 2005;46(5):781-785.

21. Mirski MA, Ferrendelli JA. Interruption of the mammillothalamic tract prevents seizures in guinea pigs. Science. 1984;226(4670):72-74.

22. Mirski MA, Tsai YC, Rossell LA, et al. Anterior thalamic mediation of experimental seizures: selective EEG spectral coherence. Epilepsia. 2003;44(3):355-365.

23. Van Gompel JJ, Klassen BT, Worrell GA, et al. Anterior nuclear deep brain stimulation guided by concordant hippocampal recording. Neurosurg Focus. 2015;38(6):E9.

24. Smeets AYJM, Duits AA, Plantinga BR, et al. Deep brain stimulation of the internal globus pallidus in refractory tourette syndrome. Clin Neurol Neurosurg. 2016;142:54-59.

25. Matias CM, Mehanna R, Cooper SE, et al. Correlation among anatomic landmarks, location of subthalamic deep brain stimulation electrodes, stimulation parameters, and side effects during programming monopolar review. Neurosurgery. 2015;11(Suppl 2):99-108.

26. Lehtimäki K, Coenen VA, Gonçalves Ferreira A, et al. The surgical approach to the anterior nucleus of thalamus in patients with refractory epilepsy: experience from the international multicenter registry (MORE). Neurosurgery. 2019;84(1): 141-150.

27. Chaturvedi A, Luján JL, McIntyre CC. Artificial neural network based characterization of the volume of tissue activated during deep brain stimulation. J Neural Eng. 2013;10(5):056023.

28. Cheung T, Noecker AM, Alterman RL, et al. Defining a therapeutic target for pallidal deep brain stimulation for dystonia. Ann Neurol. 2014;76(1):22-30.

29. Mai JK, Paxinos G, Voss T. Atlas of the Human Brain. 3rd ed. Amsterdam, The Netherlands: Elsevier; 2008.

30. Lozano AM, Lipsman N. Probing and regulating dysfunctional circuits using deep brain stimulation. Neuron. 2013;77(3):406-424.

31. Hescham S, Lim LW, Jahanshahi A, et al. Deep brain stimulation in dementiarelated disorders. Neurosci Biobehav Rev. 2013;37(10):2666-2675.

32. Mirski MA, Rossell LA, Terry JB, et al. Anticonvulsant effect of anterior thalamic high frequency electrical stimulation in the rat. Epilepsy Res. 1997;28(2):89-100.

33. Hamani C, Ewerton FIS, Bonilha SM, et al. Bilateral anterior thalamic nucleus lesions and high-frequency stimulation are protective against pilocarpine-induced seizures and status epilepticus. Neurosurgery. 2004;54(1):191-197.

34. Upton AR, Cooper IS, Springman M, Amin I. Suppression of seizures and psychosis of limbic system origin by chronic stimulation of anterior nucleus of the thalamus. Int J Neurol. 1985;19-20:223-230.

35. Hodaie M, Wennberg RA, Dostrovsky JO, Lozano AM. Chronic anterior thalamus stimulation for intractable epilepsy. Epilepsia. 2002;43(6):603-608.

36. Kerrigan JF, Litt B, Fisher RS, et al. Electrical stimulation of the anterior nucleus of the thalamus for the treatment of intractable epilepsy. Epilepsia. 2004;45(4):346354.
37. Wang $\mathrm{Y}$, Liang J, Xu C, et al. Low-frequency stimulation in anterior nucleus of thalamus alleviates kainate-induced chronic epilepsy and modulates the hippocampal EEG rhythm. Exp Neurol. 2016;276:22-30.

38. Grewal SS, Middlebrooks EH, Kaufmann TJ, et al. Fast gray matter acquisition T1 inversion recovery MRI to delineate the mammillothalamic tract for preoperative direct targeting of the anterior nucleus of the thalamus for deep brain stimulation in epilepsy. Neurosurg Focus. 2018;45(2):E6.

39. Miocinovic $S$, Somayajula S, Chitnis $S$, et al. History, applications, and mechanisms of deep brain stimulation. JAMA Neurol. 2013;70(2):163-171.

40. Stypulkowski PH, Stanslaski SR, Jensen RM, et al. Low-frequency stimulation in anterior nucleus of thalamus alleviates kainate-induced chronic epilepsy and modulates the hippocampal EEG rhythm. Brain Stimul. 2014;7(3):350-358.

41. Girgis F, Miller JP. White matter stimulation for the treatment of epilepsy. Seizure. 2016;37:28-31.

42. Forstmann BU, Isaacs BR, Temel Y. Ultra high field MRI-guided deep brain stimulation. Trends Biotechnol. 2017;35(10):904-907.

43. Rodrigues N, Mithani K, Meng Y, et al. The emerging role of tractography in deep brain stimulation: basic principles and current applications. Brain Sci. 2018;8(2):23.

44. Khan S, Wright I, Javed S, et al. High frequency stimulation of the mamillothalamic tract for the treatment of resistant seizures associated with hypothalamic hamartoma. Epilepsia. 2009;50(6):1608-1611.

45. Diemath HE, Heppner F, Enge S, Lechner H. Stereotactic anterior cingulotomy in therapy resistant generalized epilepsy. Confin Neurol. 1966;27(1):124-128.

46. Cukiert A, Baumel SW, Andreolli M, et al. Effects of corpus callosum stimulation on the morphology and frequency of epileptic bursts in the feline topical penicillin generalized model. Stereotact Funct Neurosurg. 1989;52(1):18-25.

47. Koubeissi MZ, Kahriman E, Syed TU, et al. Low-frequency electrical stimulation of a fiber tract in temporal lobe epilepsy. Ann Neurol. 2013;74(2):223-231.

48. Kros L, Eelkman Rooda OHJ, De Zeeuw CI, et al. Controlling cerebellar output to treat refractory epilepsy. Trends Neurosci. 2015;38(12):787-799.

49. Wycis HT, Baird HW, Spiegel EA. Pallidotomy and pallido-amygdalotomy in certain types of convulsive disorders. Confin Neurol. 1957;17(1):67-68.

50. Natsume J, Bernasconi N, Andermann F, et al. MRI volumetry of the thalamus in temporal, extratemporal, and idiopathic generalized epilepsy. Neurology. 2003;60(8):1296-1300.

51. Tourdias T, Saranathan M, Levesque IR, et al. Visualization of intra-thalamic nuclei with optimized white-matter-nulled MPRAGE at 7T. Neuroimage. 2014;84:534-545.

\section{Acknowledgments}

We would like to thank Cameron C. McIntyre and Angela M. Noecker from Case Western Reserve University for their kind help in calculation of the VTAs and Frans Gielen from Medtronic for helpful discussions regarding the localization of lead contacts. The authors are greatly indebted to Geertjan van Zonneveld and Artemis Iatrou for their contribution to the figures in this article and to Bethany Isaacs and Dimo Ivanov from Maastricht University for their help in acquiring the 7T MR images.

Neurosurgery Speaks! Audio abstracts available for this article at www. neurosurgeryonline.com

Supplemental digital content is available for this article at www. neurosurgery-online.com.

Supplemental Digital Content 1. Table. Group characteristics.

Supplemental Digital Content 2. Table. Patient characteristics. Abbreviations: $\mathrm{R}$; responder, NR; non-responder, VNS; vagal nerve stimulation.

Supplemental Digital Content 3. Figure. Stimulation heat maps in coronal and sagittal view with the ANT-MTT junction as the origin $[x=0, y=0, z=0]$.

Supplemental Digital Content 4. Figure. The MTT (arrow and red tracts) visualized in coronal $\mathbf{A}$, axial $\mathbf{B}$, and sagittal $\mathbf{C}$ directions by ultra-high field (UHF) MRI and diffusion weighted imaging based tractography $\mathbf{D}$. The sequence used was a T1 white-matter-nulled MPRAGE ${ }^{51}$ (voxel size: $0.8 \times 0.8 \times 0.8 \mathrm{~mm}$, $\mathrm{TE} / \mathrm{TR} / \mathrm{TI}$ of $3.3 / 4.5 / 617 \mathrm{~ms}$ ) obtained from a healthy control using a $7 \mathrm{~T}$ 
magnet (Siemens, Erlangen, Germany) and a 32-channel head coil (Nova Medical, Wilmington, Massachusetts) at the Maastricht Brain Imaging Centre. The right ANT (orange) and mammillary body (yellow) were manually segmented on $7 \mathrm{~T}$ MR images. Subsequently, probabilistic fiber tracking with FSL's probtrackx 2 was performed starting in the mammilary body using the ANT as inclusion mask and manually excluding fibers leaking into the fornix.

CNS Spotlight available at cns.org/spotlight.

\section{COMMENT}

n this manuscript, the authors present a retrospective analysis of outcomes following deep brain stimulation (DBS) of the anterior nucleus of the thalamus (ANT) - specifically focusing on the effect of stimulation location on seizure outcomes after one year of stimulation. While studies have demonstrated the long-term benefit of ANT neuromodulation, ${ }^{1}$ only a few have searched for the ideal location of stimulation or "hot spot" in the ANT. We have previously described that the specific location of stimulation may affect seizure outcome, particularly with the anatomical variability of the ANT that exists in patients with chronic epilepsy. ${ }^{2}$ Specifically, we found that stimulation more anteriorly, medially, and superiorly in the ANT was associated with greater seizure reduction. We therefore hypothesized that stimulation of the anteroventral ANT subnucleus may play a role in efficacy. Lehtimäki et $\mathrm{al}^{3}$ subsequently performed a more thorough analysis in which they similarly found that stimulation should be located more anteriorly and superiorly. While the current study also suggests that more anterior stimulation is associated with superior clinical outcomes, they also suggest the nuanced difference that specific targeting of the mammillothalamic tract (MTT) is necessary for improved rates of seizure reduction.

While this study serves as the largest series to date focused on identifying the optimal target of stimulation within the ANT, further studies are still necessary to further investigate these preliminary findings. Overall, I believe that this work significantly adds to our current understanding regarding the correct location of stimulation within the ANT in DBS for epilepsy.

Chengyuan Wu Philadelphia, Pennsylvania

1. Salanova V, Witt T, Worth R, et al. Long-term efficacy and safety of thalamic stimulation for drug-resistant partial epilepsy. Neurology. 2015;84(10):1017-1025.

2. Wu C, D'Haese PF, Pallavaram S, et al. Variations in Thalamic Anatomy Affect Targeting in Deep Brain Stimulation for Epilepsy. Stereotact Funct Neurosurg. 2016;94(6):387-396.

3. Lehtimäki K., Möttönen T, Järventausta K, et al. Outcome based definition of the anterior thalamic deep brain stimulation target in refractory epilepsy. Stereotact Funct Neurosurg. 2016;9(2):268-275. 